\title{
Challenges relating to comparison of flavonoid glycosides dissolution profiles from Sutherlandia frutescens products
}

\author{
OLUCHI N MBAMALU ${ }^{1, *}$ \\ JAMES SYCE ${ }^{1,2}$ \\ HALIMA SAMSODIEN ${ }^{3}$ \\ ${ }^{1}$ Discipline of Pharmacology \\ School of Pharmacy \\ University of the Western Cape \\ Private Bag X17, Bellville 7535 \\ Cape Town, South Africa \\ ${ }^{2}$ South African Herbal Sciences \\ and Medicines Institute \\ University of the Western Cape \\ Private Bag X17, Bellville 7535 \\ Cape Town, South Africa \\ ${ }^{3}$ Discipline of Pharmaceutics \\ School of Pharmacy, University \\ of the Western Cape, Private Bag X17 \\ Bellville 7535, Cape Town \\ South Africa \\ Accepted August 3, 2016 \\ Published online September 7, 2016
}

\begin{abstract}
Unlike the case of conventional drug formulations, dissolution tests have hitherto not been required for herbal medicinal products commercially available in South Africa. This study investigated dissolution of the South African Sutherlandia frutescens using selected flavonoid glycosides as marker compounds. Dissolution of markers was assessed in three dissolution media at $\mathrm{pH} 1.2,4.5$ and 6.8, and samples were analysed using a validated HPLC method. The dissolution profile of each marker varied for the different materials investigated. All three media utilised showed differences in flavonoid glycoside dissolution between the S. frutescens products evaluated, with $f_{2}$ values $<50$ for comparison of flavonoid dissolution from any two of the materials. Dissolution of $S$. frutescens materials could thus be characterised using the markers in all the media tested. This tool may be employed in the future for comparison of orally administered $S$. frutescens products, provided between-batch variability is evaluated and found less than between-sample variability.
\end{abstract}

Keywords: Sutherlandia frutescens, herbal products, dissolution testing, flavonoid, discriminatory medium, similarity factor

There is a global need for regulation and quality assurance of herbal medicinal products (1). In general, the tools and experience to effectively meet this need are lacking especially in many developing countries. This also applies to popular and widely used indigenous South African herbal medicinal products such as Sutherlandia frutescens. It is traditionally reputed to be a cure for many ailments, and some of its claimed pharmacological effects have been proven by in vitro tests (2). It has recently gained renewed interest due to reports from patients and proponents of herbal medicine therapy on its activity against HIV, and concerns regarding interactions when co-administered with antiretroviral drugs $(2,3)$. Consequently, arrangements are underway for a phase I pharmacokinetic study to assess the bioavailability of $S$. frutescens products. In such studies, $S$. frutescens

\footnotetext{
*Correspondence; e-mail: ombamalu@uwc.ac.za
} 
products of established and consistent pharmaceutical quality would be required. One such quality is the release of its active constituents in dissolution tests, for which a discriminatory medium and appropriate marker compounds should be employed.

Flavonoids have been reported as constituents of $S$. frutescens (4) and may contribute to the activity of this plant medicine (2). In S. frutescens, flavonoid glycosides (sutherlandins A, B, C and D) of the aglycones, quercetin and kaempferol, have been reported as possible chemical markers (4), and may serve as suitable references for comparison and in vitro dissolution tests.

Crucial to mention though is the fact that selection of marker compounds for dissolution tests or otherwise of herbal materials such as S. frutescens will pose challenges. Firstly, unlike conventional drug formulations where activity is attributed to a single known and well researched compound, herbal medicinal products (HMPs) are made up of multiple components, some of which may not have a direct effect on pharmacological properties of the product. Secondly, the ratio of these different constituents may vary according to cultivation, harvesting or processing methods, influencing the in vitro release and hence efficacy of the compound (5). While rigorous quality control tests may not be required for HMPs, because they may be classified as dietary supplements and not as drugs, efforts are made to improve the quality of such products in many countries such as South Africa and Croatia, to mention just a few $(6,7)$. Such quality improvement measures may involve dissolution studies for comparison of products. Thus far, no method has been documented for the evaluation of in vitro dissolution profiles of $S$. frutescens products.

In this study, a simple modified pharmacopoeial dissolution method is employed for S. frutescens products. The results from this study are discussed within the context of differentiation between materials of the popular S. frutescens.

\section{EXPERIMENTAL}

\section{Reagents and samples}

Samples of S. frutescens leaf powder (LP, batch number: BN E16794; Afriplex, Paarl, South Africa), spray-dried aqueous extract 1 (SDAE 1; batch number: Ferl-DST/001-1210, South Africa), and spray-dried aqueous extract 2 (SDAE 2, batch number: 1674; Ferlot Manufacturing \& Packaging Pty Ltd, South Africa) were used. Their flavonoid glycosides (sutherlandins A, B, C and D), not commercially available, were isolated from the $n$-butanol soluble portion of the ethyl acetate extract of $S$. frutescens spray-dried aqueous extract (SDAE) material. The butanol extract was subjected to reversed phase column chromatography using solid phase extraction (SPE) cartridges for the isolation of flavonoid fractions, from which flavonoid glycoside compounds were isolated. Acetonitrile (HPLC grade) was from Sigma Aldrich, while formic acid (analytical grade) and concentrated hydrochloric acid (analytical grade) were from Merck. All water used was obtained from an in-house Milli-Q purification system (Millipore, USA).

\section{Instrumentation}

Dissolution tests were conducted using the USP basket-type apparatus (VanKel VK 700 , USA) at $37 \pm 0.5^{\circ} \mathrm{C}$. Stainless steel baskets of size 40 were used, and the baskets were 
rotated at 100 revolutions per minute. Analysis of marker compounds in dissolution media was conducted using a validated HPLC method on an Agilent 1200 series HPLC system equipped with an in-line degassing system, quaternary pump, auto-loading sampler, thermostatted column compartment and photodiode array detector.

\section{Chromatographic conditions}

Analysis of marker compounds in dissolution media was conducted using an Agilent 1200 series HPLC system equipped with an in-line degassing system, quaternary pump, auto-loading sampler, thermostatted column compartment and photodiode array detector, as previously described (8). Chromatographic separation was obtained on a Phenomenex Luna ${ }^{\circledR} \mathrm{C}_{18}$ column $(25 \mathrm{~cm} \times 4.6 \mathrm{~mm}, 5 \mu \mathrm{m}$ i.d. $)$ with a compatible guard column, both maintained at $45{ }^{\circ} \mathrm{C}$. The mobile phase consisted of water $(0.01 \%$ formic acid) (A) and acetonitrile (0.01\% formic acid) (B), filtered and degassed prior to use. The flow rate of the mobile phase was maintained at $0.8 \mathrm{~mL} \mathrm{~min}^{-1}$, injection volume was $20 \mu \mathrm{L}$, and peaks were separated by gradient elution: 0 to $1 \mathrm{~min}, 82 \% \mathrm{~A} / 18 \% \mathrm{~B} ; 1$ to $15 \mathrm{~min}, 82 \% \mathrm{~A} / 18 \% \mathrm{~B}$ to $75 \% \mathrm{~A} / 25$ $\% \mathrm{~B} ; 15$ to $20 \mathrm{~min}, 75 \% \mathrm{~A} / 25 \% \mathrm{~B}$ to $65 \% \mathrm{~A} / 35 \% \mathrm{~B} ; 20$ to $25 \mathrm{~min}, 65 \% \mathrm{~A} / 35 \% \mathrm{~B}$ to $40 \% \mathrm{~A} /$ $60 \% \mathrm{~B} ; 25$ to $26 \mathrm{~min}, 40 \% \mathrm{~A} / 60 \% \mathrm{~B}$ to $82 \% \mathrm{~A} / 18 \% \mathrm{~B}$, followed by equilibration with $82 \%$ A/18 \% B from 26 to 35 minutes. The eluent was monitored at several wavelengths over a range from 250 to $380 \mathrm{~nm}$; the specific wavelength of interest was $370 \mathrm{~nm}$. Data acquisition and processing were accomplished using the OpenLAB ${ }^{\mathrm{TM}}$ CDS ChemStation Edition software.

\section{Encapsulation of S. frutescens materials}

The $S$. frutescens materials used (LP, SDAE 1 and SDAE 2) were filled into size 0 capsules using a Cap-M-Quik ${ }^{\mathrm{TM}}$ capsule filler (S L Sanderson \& Co., Berry Creek, CA, USA). Capsules were weighed before and after filling in order to calculate the weight of the encapsulated material and assess variation.

\section{Validation of dissolution method}

The dissolution test used in this study is classified as a category III procedure (i.e., analytical procedure for determination of performance characteristics); hence the need to evaluate its precision by repeatability (9). Six replicate samples, containing the SDAE 1 material, were collected from six vessels of the dissolution apparatus (at $\mathrm{pH} 1.2$ ) at a 60-minute sampling time. The samples were analysed to validate method precision, and the values were expressed as relative standard deviation (\% RSD) between samples.

\section{Dissolution conditions}

In line with dissolution guidelines for new products, dissolution tests were conducted in three dissolution media at $\mathrm{pH} 1.2,4.5$ and 6.8 , all maintained at $37 \pm 0.5{ }^{\circ} \mathrm{C}(10)$. Although the dissolution medium volume of $900 \mathrm{~mL}$ is commonly used in dissolution tests, reduction of this volume may be advocated in cases where the label claim is less than $5 \mathrm{mg}$ (11). Due to the low level of marker compounds in the S. frutescens materials studied (i.e. $<5 \mathrm{mg} / \mathrm{capsule}$ ), the official dissolution conditions were modified to $450 \mathrm{~mL}$ of dissolution medium for each 
vessel. In each of the twelve replicate vessels of the dissolution apparatus containing the appropriate dissolution medium at either $\mathrm{pH} 1.2,4.5$ or 6.8 , two capsules containing the $S$. frutescens product to be evaluated were introduced into mesh baskets used to retain the capsules and the baskets were rotated at 100 revolutions per minute (rpm). At predetermined time intervals $(5,10,15,20,30,60,90$ and 120 minutes), $3 \mathrm{~mL}$ of sample was withdrawn from each vessel through a 0.45 micron nylon membrane and replaced with an equal volume of the same pre-warmed medium in order to maintain a constant volume.

\section{Data analysis}

Withdrawn samples were analysed using a validated HPLC method. The amount of each marker (flavonoid glycoside) compound dissolved at specified sampling times was calculated from the peak areas of the HPLC chromatograms. Cumulative percentage released was calculated from the ratio of the quantified amount to the average flavonoid content in capsules. Data was plotted as percentage dissolved \pm standard deviation (SD) vs. sample dissolution time. Dissolution profiles were compared using the similarity factor, $f_{2}(12)$.

\section{RESULTS AND DISCUSSION}

Following encapsulation, the masses obtained for the encapsulated LP, SDAE 1 and SDAE 2 materials were $322.5 \pm 8.7,324.9 \pm 7.6$ and $414.8 \pm 9.1 \mathrm{mg}$ (mean \pm SD), respectively.

The dissolution method precision validation returned values of $4.4,4.8,2.7$ and $17.0 \%$ for sutherlandins A, B, C and D, respectively. Dissolution profiles of the assessed flavonoids from $S$. frutescens LP, SDAE 1 and SDAE 2 are presented in Fig. 1 to 4 . The figures show the release of each sutherlandin from $S$. frutescens LP, SDAE 1 and SDAE 2 under three different $\mathrm{pH}$ conditions: $1.2,4.5$ and 6.8. Table I shows the $f_{2}$ values for comparison of flavonoid dissolution from the $S$. frutescens LP, SDAE 1 and SDAE 2 materials.
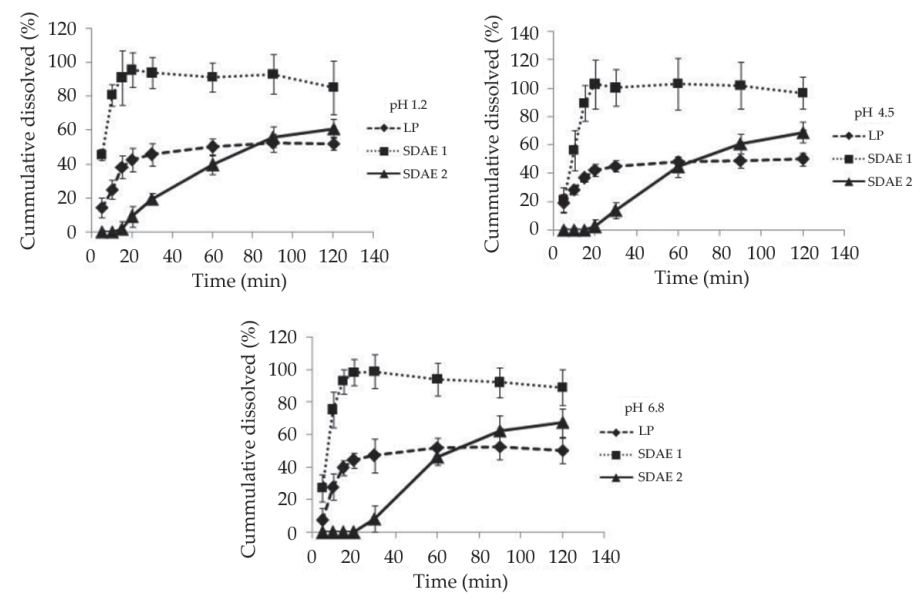

Fig. 1. Dissolution profiles of sutherlandin A from S. frutescens products at $\mathrm{pH} 1.2,4.5$ and 6.8 (mean $\pm \mathrm{SD}$ ). LP - leaf powder, SDAE - spray-dried aqueous extract. 
Masses obtained for the encapsulated LP, SDAE 1 and SDAE 2 materials were $322.5 \pm 8.7$, $324.9 \pm 7.6$ and $414.8 \pm 9.1 \mathrm{mg}$ (mean $\pm \mathrm{SD}$ ), respectively. This complied with the pharmacopoeial requirements for weight uniformity, i.e., between 85 and $115 \%$ of the mean.

The dissolution method showed precision for the quantification of three out of four flavonoid glycosides (sutherlandins A, B and C) calculated from samples withdrawn from the dissolution medium (at $\mathrm{pH} 1.2$ ) at the 60 -minute sampling time. The \% RSD values for
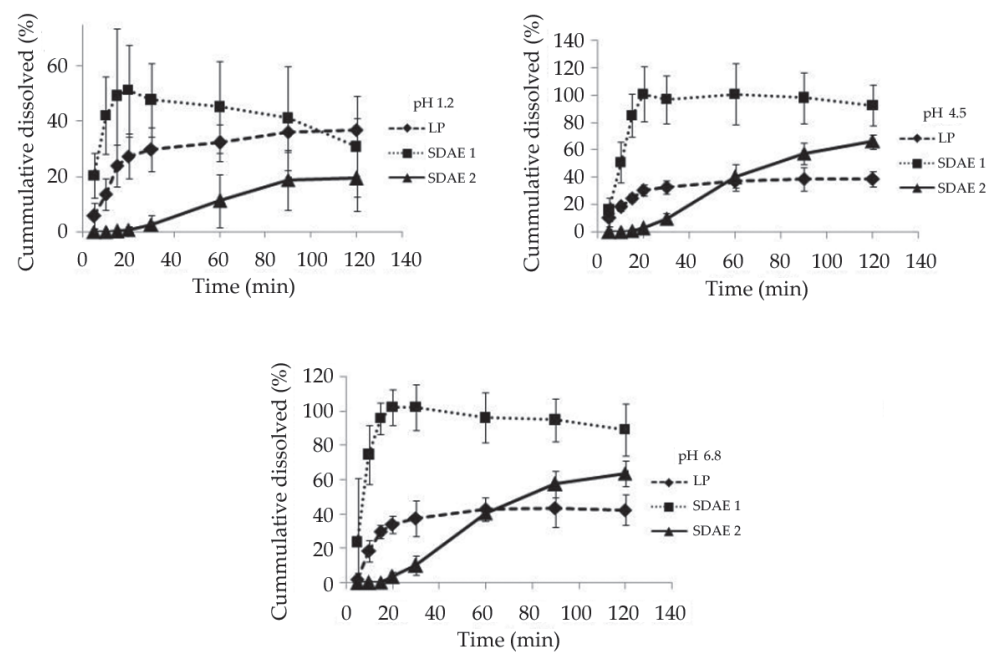

Fig. 2. Dissolution profiles of sutherlandin B from S. frutescens products at $\mathrm{pH} 1.2,4.5$ and 6.8 (mean \pm SD). LP - leaf powder, SDAE - spray-dried aqueous extract.
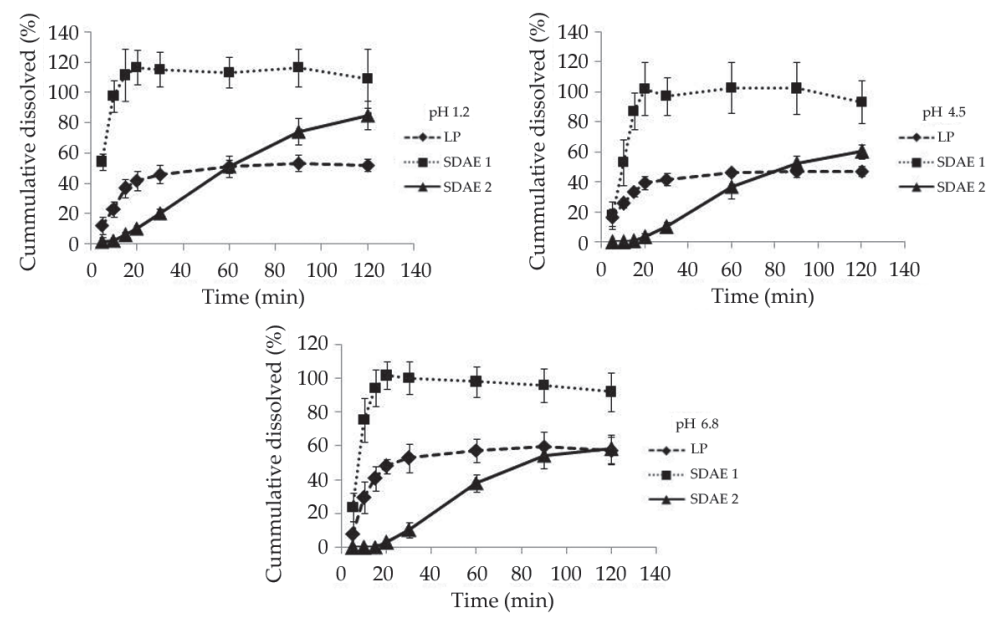

Fig. 3. Dissolution profiles of sutherlandin C from S. frutescens products at $\mathrm{pH} 1.2,4.5$ and 6.8 (mean $\pm \mathrm{SD}$ ). LP - leaf powder, SDAE - spray-dried aqueous extract. 

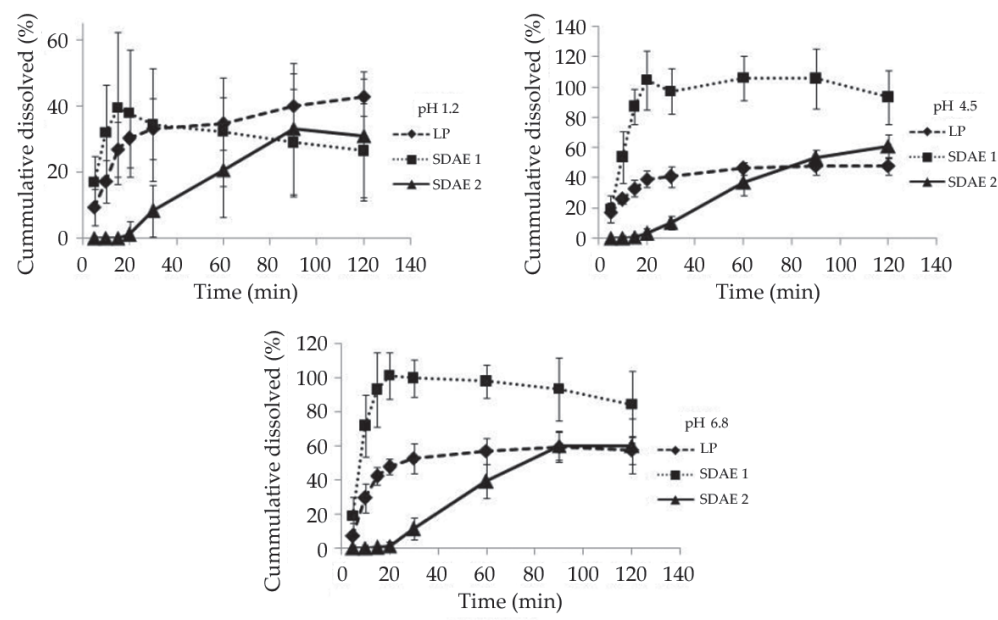

Fig. 4. Dissolution profiles of sutherlandin D from S. frutescens products at $\mathrm{pH} 1.2,4.5$ and 6.8 (mean \pm SD). LP - leaf powder, SDAE - spray-dried aqueous extract.

sutherlandins A, B and C were 4.4, 4.8 and $2.7 \%$, respectively, all within the specified criterion of $\leq 5 \%$ (9). This suggests that the dissolution method described herein is precise for the analysis of sutherlandins A, B and C from the different S. frutescens materials. The $\%$ RSD for sutherlandin D was however $17.0 \%$, outside the specification limits, possibly due to degradation of this marker compound at different rates in the different vessels containing an acidic dissolution medium. This may imply that sutherlandin D may not be a good marker compound for the analysis of $S$. frutescens plant materials at $\mathrm{pH}$ 1.2. However, such conclusions cannot be drawn regarding its suitability at $\mathrm{pH} 4.5$ and 6.8 (or in any other suitable dissolution medium). In addition, the fact that sutherlandins A, B and C seem suitable for dissolution profile comparison at $\mathrm{pH} 1.2$ does not imply that they are suitable for dissolution tests in other suitable dissolution media. Any inference regarding the suitability of any marker in any medium may only be drawn when suitable validation tests are performed in advance.

Perusal of Figs. 1 to 4 shows that dissolution of sutherlandins A, B, C and D from all three assessed products varied from delayed to rapidly dissolving. From the LP products under all three $\mathrm{pH}$ conditions, none of the marker compounds displayed the characteristic of immediate release (i.e., at least $75 \%$ dissolved within 45 minutes). From SDAE 2 under all three $\mathrm{pH}$ conditions, all the marker compounds (except sutherlandin $\mathrm{C}$ at $\mathrm{pH}$ 1.2) displayed a lag time of at least 10 minutes prior to dissolution. In all three media tested, dissolution from SDAE 1 was the fastest while dissolution from SDAE 2 was the slowest.

With respect to origin, the LP product consists of small bits of aerial S. frutescens plant materials, from which SDAE 1 and SDAE 2 products were made (not necessarily the same $\mathrm{LP}$ batch investigated here). Being aqueous extracts, it was presumed that dissolution rates of the sutherlandins from SDAE 1 and SDAE 2 would be faster than from LP. The results (Figs. 1 to 4) however show faster marker compound dissolution from LP and SDAE 1 products compared to SDAE 2. It seems that the aqueous extract nature of SDAE 2 was not 
sufficient to hasten flavonoid glycoside dissolution from this product when compared to LP. The LP, though not an aqueous extract, exhibited faster dissolution than SDAE 2. Some other characteristic over-riding its non-aqueous nature may have contributed to such an effect. Previous studies have reported the presence of polysaccharides in S. frutescens leaves (13), and so further testing of the three $S$. frutescens products for sugars and starch were conducted using Benedict's and iodine tests, respectively. The presence of starch was detected in SDAE 2. While starch is generally used as a disintegrant in solid dose formulations, it may also serve as a binder or anti-adherent and, in such cases, may retard marker compound dissolution from a product. This may account for the slower dissolution of sutherlandins from SDAE 2. The LP and SDAE 1 materials did not test positive for starch; however, the presence of sugars was noted (assessed by precipitate formation and colour), more concentrated in the LP material than in aqueous extracts. This could account for the faster dissolution of LP compared to SDAE 2. SDAE 2 is considered an older product than SDAE 1 (i.e., SDAE 2 was produced earlier than SDAE 1). Is there perhaps a possibility that the 'unprocessed' and more recently processed S. frutescens materials (e.g., LP or SDAE 1) contain more sugar and little or no starch, with the sugar converted to starch over time? Could this explain why the more recent batches contained traces of simple sugar and no starch while the older product (SDAE 2) contained more starch? However, no confirmation of this has been found in literature.

At $\mathrm{pH}$ values of 4.5 and 6.8, all the flavonoid glycosides displayed immediate release characteristics from SDAE 1. At $\mathrm{pH}$ 1.2, however, only sutherlandins A and C from SDAE 1 displayed such characteristics. Dissolution of sutherlandins B and D from SDAE 1 at $\mathrm{pH}$ 1.2 was much slower than that obtained for sutherlandins A and C. For sutherlandins B and $\mathrm{D}$ from SDAE 1 at $\mathrm{pH} 1.2$, the dissolution curve of these marker compounds displayed a downward trend after attaining maximum dissolution, while this was not the case of sutherlandins A and C. Sutherlandins B and D are different in that they are flavonoid glycosides of the aglycones, quercetin and kaempferol, respectively, while the presence of the apiofuranosyl group is a similarity (4). It is very likely that the observed decrease in percentage dissolved after peak dissolution (for sutherlandins B and D) may be caused by degradation of the flavonoid glcycoside, possibly attributable to the glycoside component of the compound. Likewise, sutherlandins $\mathrm{A}$ and $\mathrm{C}$ are different in that they are flavonoid glycosides of the aglycones, quercetin and kaempferol, respectively, while the presence of the xylopyranosyl group is a similarity (4). It is also very likely that the resistance of sutherlandins $\mathrm{A}$ and $\mathrm{C}$ to breakdown in the dissolution medium at $\mathrm{pH} 1.2$ can be attributed to this similarity - the xylopyranosyl group. The downward trend in dissolution profiles of sutherlandins $\mathrm{B}$ and $\mathrm{D}$ at $\mathrm{pH} 1.2$ was not obtained under other $\mathrm{pH}$ conditions or from the LP and SDAE 2 materials. It seems that while the sugar moiety in sutherlandins B and D may make them prone to degradation at $\mathrm{pH} 1.2$, resulting in a decrease in the cumulative amount of sutherlandins B and D dissolved over time, the SDAE 1 material also provided an enabling environment for this to occur.

Comparison of dissolution profiles for each marker compound of any two of the three S. frutescens products using the similarity factor gave values lower than 50 in all cases (Table I).

This implies dissimilarity of the assessed dissolution profiles. However, the variances obtained with the dissolution data for flavonoid glycosides were beyond the limits stipulated for the $f_{2}$ equation (i.e., $\% \mathrm{CV}<20 \%$ for earlier time points and $<10 \%$ for later time 
O. N. Mbamalu et al.: Challenges relating to comparison of flavonoid glycosides dissolution profiles from Sutherlandia frutescens products, Acta Pharm. 67 (2017) 137-146.

Table I. Comparison of $f_{2}$-values for sutherlandin dissolution profiles at $p H 1.2,4.5$ and 6.8

\begin{tabular}{|c|c|c|c|c|c|}
\hline \multicolumn{3}{|c|}{ Sutherlandin A } & \multicolumn{3}{|c|}{ Sutherlandin B } \\
\hline \multicolumn{3}{|c|}{ a) LP vs. SDAE materials } & \multicolumn{3}{|c|}{ a) LP vs. SDAE materials } \\
\hline $\mathrm{pH}$ & $f_{2}$ & $w t f_{2}$ & $\mathrm{pH}$ & $f_{2}$ & $w t f_{2}$ \\
\hline 1.2 & 17.2 & 4.9 & 1.2 & 36.3 & 17 \\
\hline 4.5 & 16 & 3.3 & 4.5 & 12.9 & -2.5 \\
\hline 6.8 & 17.5 & 4.6 & 6.8 & 12.9 & -2.2 \\
\hline \multicolumn{3}{|c|}{ b) LP vs. FDAE materials } & \multicolumn{3}{|c|}{ b) LP vs. FDAE materials } \\
\hline $\mathrm{pH}$ & $f_{2}$ & $w t f_{2}$ & $\mathrm{pH}$ & $f_{2}$ & $w t f_{2}$ \\
\hline 1.2 & 32.1 & 9.4 & 1.2 & 34.6 & 21.3 \\
\hline 4.5 & 28.8 & 4.6 & 4.5 & 34.1 & 12.9 \\
\hline 6.8 & 27.5 & 11 & 6.8 & 33.7 & 9.6 \\
\hline \multicolumn{3}{|c|}{ c) SDAE vs. FDAE materials } & \multicolumn{3}{|c|}{ a) SDAE vs. FDAE materials } \\
\hline $\mathrm{pH}$ & $\mathrm{f}_{2}$ & $w t f_{2}$ & $\mathrm{pH}$ & $f_{2}$ & $w t f_{2}$ \\
\hline 1.2 & 9.2 & -11 & 1.2 & 21.5 & 6.5 \\
\hline 4.5 & 8.9 & -16.3 & 4.5 & 9.5 & -14.6 \\
\hline 6.8 & 8.5 & -6.5 & 6.8 & 7.9 & 15.1 \\
\hline \multicolumn{3}{|c|}{ Sutherlandin C } & \multicolumn{3}{|c|}{ Sutherlandin D } \\
\hline \multicolumn{3}{|c|}{ a) LP vs. SDAE materials } & \multicolumn{3}{|c|}{ a) LP vs. SDAE materials } \\
\hline $\mathrm{pH}$ & $f_{2}$ & $w t f_{2}$ & $\mathrm{pH}$ & $f_{2}$ & $w t f_{2}$ \\
\hline 1.2 & 9.2 & -3.0 & 1.2 & 48.9 & 28.1 \\
\hline 4.5 & 15.6 & 2.9 & 4.5 & 15 & 0.6 \\
\hline 6.8 & 18.5 & 6 & 6.8 & 19.6 & 5.7 \\
\hline \multicolumn{3}{|c|}{ b) LP vs. FDAE materials } & \multicolumn{3}{|c|}{ b) LP vs. FDAE materials } \\
\hline $\mathrm{pH}$ & $f_{2}$ & $w t f_{2}$ & $\mathrm{pH}$ & $f_{2}$ & $w t f_{2}$ \\
\hline 1.2 & 30.8 & 15.8 & 1.2 & 35.8 & 5.7 \\
\hline 4.5 & 30.9 & 9.1 & 4.5 & 31.2 & 8.7 \\
\hline 6.8 & 26.7 & 3.5 & 6.8 & 26.6 & -3.3 \\
\hline \multicolumn{3}{|c|}{ c) SDAE vs. FDAE materials } & \multicolumn{3}{|c|}{ c) SDAE vs. FDAE materials } \\
\hline $\mathrm{pH}$ & $f_{2}$ & $w t f_{2}$ & $\mathrm{pH}$ & $f_{2}$ & $w t f_{2}$ \\
\hline 1.2 & 5.2 & -10.5 & 1.2 & 30 & 0.3 \\
\hline 4.5 & 8.6 & -13.8 & 4.5 & 8.3 & -14.4 \\
\hline 6.8 & 7.7 & -14.0 & 6.8 & 8.3 & -20.6 \\
\hline
\end{tabular}

$f_{2}$ - original similarity factor; $w t f_{2}$ - similarity factor modified to allow for the impact of within-batch variability on $f_{2}$

points), which may be due to the inherent variability of natural products (14). Extensive between-batch variations of the tested products may result in the overlap of dissolution profiles from the tested batches, returning erroneous values for dissolution profile com- 
parison. It may therefore be necessary to evaluate such between-batch variability prior to assessing different products in order to show that tested products can be distinguished even if different batches of respective products are used.

To address extensive within-batch variation, which may introduce errors during comparison, dissolution profiles may also be compared using a modification of the original similarity factor proposed by Gohel et al. (15) (Table I, $w t f_{2}$ ). This modification of the similarity factor, unlike the original $f_{2}$ formula, incorporates a weighting scheme to accommodate for within-batch variations, and is thought to result in a more discriminating value for dissolution profile comparison of samples with extensive variation. A more discriminating method for dissolution profile comparison in this study is defined as one with a lower $f_{2}$ value.

Further comparisons of sutherlandins A, B, C and D dissolution profiles using the modified $f_{2}$ equation all gave values below 50 , further confirming dissimilarity of dissolution profiles (Table I). In all comparison scenarios, Gohel's modified similarity factor $\left(w t f_{2}\right.$, Table I) proved to be a more discriminating tool for dissolution profile comparison, since it returned the lowest $f_{2}$ values. The $w t f_{2}$ values were also significantly different from the original $f_{2}$ values $(p<0.001)$. Dissolution of the aglycones, quercetin and kaempferol, though detected from some products and under some $\mathrm{pH}$ conditions, could not be quantified. This may be due to their very low content in the S. frutescens products ( 0 to $0.26 \%)$.

A suitable dissolution test should be able to differentiate between products that have been modified via formulation or process changes, though such modifications may be subtle. The findings from our study indicate that the dissolution test reported herein can be used to differentiate between the $S$. frutescens products studied. For the test, flavonoid glycosides were found to be better marker and differentiating compounds than flavonoid aglycones for dissolution studies of $S$. frutescens materials; they were detected in the different dissolution media and also projected different dissolution properties for different $S$. frutescens materials. Glycosylation of a flavonoid decreases its reactivity while improving its water solubility (16), enhancing its dissolution in water-based media. This may serve to explain the detection of flavonoid glycosides, to different degrees, in the dissolution media assessed. This may also be due to the content of the flavonoid glycoside, which is more than that of the flavonoid aglycones in water-based extracts of the different products.Dissolution testing of $S$. frutescens materials therefore enabled release of flavonoid glycosides in the three dissolution media tested, and each of the three media could be used to differentiate between the S. frutescens materials studied. In acidic medium (at $\mathrm{pH} 1.2)$, however, it may be preferable to employ only sutherlandins $\mathrm{A}$ and $\mathrm{C}$ as marker compounds. Their dissolution profiles were not adversely affected by acidic $\mathrm{pH}$, and no breakdown of these compounds was noticed in any of the dissolution media assessed.

Dissolution characteristics of each flavonoid compound from the different $S$. frutescens materials show that the same flavonoid glycoside may not necessarily be released from different materials at the same rate. This may be due to the differences in their release mechanisms from different materials, which is currently under investigation. Such differences may also translate to differences in in vivo bioavailability of flavonoid compounds from the different materials.

It is crucial to mention that whilst flavonoid glycosides have been identified in S. frutescens as potential marker compounds, and display some properties associated with its potential medicinal activities, there is as yet no clear indication that they are the major 
active components. Therefore, although their dissolution profiles will provide a means of comparing extracts, changes in dissolution profiles may not necessarily reflect the efficacy of a given extract.

Acknowledgements. - The authors thank the Department of Science and Technology (grant number DST/ICON 167/2010, dated 4 November 2010 and revised 10 Oct 2013) for funding assistance.

\section{REFERENCES}

1. P. O. Erah, Herbal medicines: challenges, Trop. J. Pharm. Res. 1 (2002) 53-54.

2. D. R. Katerere and J. N. Eloff, Antibacterial and antioxidant activity of Sutherlandia frutescens (Fabaceae), a reputed anti-HIV/AIDS phytomedicine, Phytother. Res. 19 (2005) 779-781; DOI: 10.1002/ ptr.1719.

3. P. S. Fasinu, H. Gutmann, H. Schiller, A. D. James, P. J. Bouic and B. Rosenkranz, The potential of Sutherlandia frutescens for herb-drug interaction, Drug Metab. Dispos. 41 (2013) 488-497; DOI: 10.1124/dmd.112.049593.

4. X. Fu, X. C. Li, Y. H. Wang, B. Avula, T. J. Smillie, W. Mabusela, J. Syce, Q. Johnson, W. Folk and I. A. Khan, Flavonol glycosides from the South African medicinal plant Sutherlandia frutescens, Planta Med. 76 (2010) 178-181; DOI: 10.1055/s-0029-1186030.

5. P. Jin, S. Madieh and L. L. Augsburgers, Challenges with dissolution testing and quality assessment for commercial feverfew products, Dissolut. Technol. (2007) 14-20; DOI: 10.14227/DT140307P14.

6. G. Gabriels, M. Lambert, P. Smith and D. Hiss, Will the new Consumer Protection Act prevent harm to nutritional supplement users?, S. Afr. Med. J. 101 (2011) 543-545.

7. M. Vujiić and L. Pollak, Composition, labelling, and safety of food supplements based on bee products in EU legislation - Croatian experiences, Arh. Hig. Toksikol. 66 (2015) 243-249; DOI: 10.1515/aiht-2015-66-2654.

8. O. N. Mbamalu, E. Antunes, N. Silosini, H. Samsodien and J. Syce, HPLC determination of selected flavonoid glycosides and their corresponding aglycones in Sutherlandia frutescens materials, Med. Arom. Plants. 5 (2016); DOI: doi:10.4172/2167-0412.1000246.

9. A. R. T. Costa, S. F. Alves, E. C. da Conceição, C. F. D. Garrote, J. R. Paula and M. T. F. Bara, Dissolution test of herbal medicines containing Passiflora sp., Braz. J. Pharmacogn. 21 (2011) 525-531; DOI: 10.1590/S0102-695X2011005000083.

10. O. Anand, L. X. Yu, D. P. Conner and B. M. Davit, Dissolution testing for generic drugs: an FDA perspective, AAPS J. 13 (2011) 328-335; DOI: 10.1208/s12248-011-9272-y.

11. S. P. Karuppiah, Analytical method development for dissolution release of finished solid oral dosage forms, Int. J. Curr. Pharm. Res. 4 (2012) 48-53.

12. J. W. Moore and H. H. Flanner, Mathematical comparison of dissolution profiles, Pharma Tech. 20 (1996) 64-74.

13. B. Zhang, W. K. Leung, Y. Zou, W. Mabusela, Q. Johnson, T. E. Michaelsen and B. S. Paulsen, Immunomodulating polysaccharides from Lessertia frutescens leaves: isolation, characterization and structure activity relationship, J. Ethnopharmacol. 152 (2014) 340-348.

14. V. K. R. Ghosh, S. G. Bhope, V. V. Kuber, P. S. Gaikwad and M. J. Patil, Development and validation of dissolution test method for andrographolide from film coated polyherbal tablet formulation, Int. J. Phar. Pharm. Sci. 4 (2012) 307-312.

15. M. C. Gohel, K. G. Sarvaiya, A. R. Shah and B. K. Brahmbhatt, Mathematical approach for the assessment of similarity factor using a new scheme for calculating weight, Indian J. Pharm. Sci. 71 (2009) 142-144; DOI: 10.4103/0250-474X.54281.

16. S. Kumar and A. K. Pandey, Chemistry and biological activities of flavonoids: an overview, Sci. World J. (2013) DOI: 10.1155/2013/162750. 